\title{
Letter to the Editor on "Socially Distant Medical Education in the Face of COVID-19"
}

\author{
Shreyas Moodalbyle ${ }^{1} \cdot$ Devika Nair $^{1}$
}

Accepted: 30 July 2021 / Published online: 11 August 2021

(C) International Association of Medical Science Educators 2021

\section{Dear Editor,}

We sincerely thank Vasavda et al. for their commentary, which discusses the rapid restructuring of the medical curriculum to a virtual medium due to the COVID-19 pandemic [1]. As medical students who were on the forefront of this transition, we would like to offer our perspectives on its strengths and weaknesses.

We agree that the main benefit of virtual education is flexibility, as any student with a stable internet connection can view the educational content from the comfort of their home. This also removes travelling times and costs, a welcome benefit in an increasingly environment-conscious world. Whilst we understand that this may lead to some students feeling "detached" from the education faculty, in our own experiences, education faculty members have remained largely accessible through email and regular virtual meetings, and in some instances, communication between students and faculty has been improved [1].

The advantages of virtual education were especially seen during lockdown periods of 2020, when clinical placements and in-person lectures were not possible. During this time, various groups emerged to continue medical education freeof-charge, for example, the group Sustaining Medical education In a Lockdown Environment (SMILE) [2]. Furthermore, existing literature documents that virtual education is generally well-received by medical students and can be beneficial in improving learning outcomes [3].

We would like to thank the authors for highlighting that virtual education may "further the digital divide" and have potential impacts on students' mental health due to a lack of social interaction. Other potential weaknesses of virtual teaching include the inability to emulate clinical experiences gained from hospital environments, and the dearth of evidence-based processes that can be implemented in order to transition from a traditional teaching model to a digital model [4].

Shreyas Moodalbyle

sxm1287@student.bham.ac.uk

1 College of Medical and Dental Sciences, University of Birmingham, Birmingham, UK
Another issue highlighted by the commentary is the lack of institutional infrastructure in virtual education. Whilst lecturers have improved their ability to teach virtually as the pandemic progresses, central information technology support teams and tutorials on how to navigate teleconferencing software would benefit all parties involved in virtual education. Institutions could perhaps utilise the lower operational costs of virtual education compared to in-person education to fund such support systems.

To conclude, we argue that there is a place for e-lectures and virtual small group sessions in future medical curricula. As further waves of the pandemic and social distancing measures threaten clinical placements, additional research is required so that medical institutions can ensure high-quality teaching is maintained throughout these unprecedented times.

\section{Declarations}

Conflict of Interest The authors declare no competing interests.

\section{References}

1. Vasavda C, Ho BK, Davison A. Socially distant medical education in the face of COVID-19. Medical Science Educator. 2021.

2. SMILE: Sustaining Medical education In a Lockdown Environment [Internet]. [cited $2020 \mathrm{Jul} 24$ ]. Available from: https://www. facebook.com/groups/257148981982669/about/

3. Tang B, Coret A, Qureshi A, Barron H, Ayala AP, Law M. Online lectures in undergraduate medical education: scoping review [Internet]. Vol. 20, Journal of Medical Internet Research. J Med Internet Res. 2018 [cited 2020 Jul 24]. Available from: https:// pubmed.ncbi.nlm.nih.gov/29636322/

4. Tang B, Coret A, Barron H, Qureshi A, Law M. Online lectures in undergraduate medical education: how can we do better? Can Med Educ J [Internet]. 2019 Mar 14 [cited 2020 Jul 24];10(1):e137139. Available from: http://www.cmej.ca

Publisher's Note Springer Nature remains neutral with regard to jurisdictional claims in published maps and institutional affiliations. 